\title{
An association between non-gonococcal urethritis and bacterial vaginosis and the implications for patients and their sexual partners
}

\author{
F E A Keane, B J Thomas, L Whitaker, A Renton, D Taylor-Robinson
}

Objectives: The aetiology of non-gonococcal urethritis (NGU) in a considerable proportion of men remains unaccounted for. We wished to investigate the possible aetiological role of bacterial vaginosis (BV), the commonest cause of abnormal discharge in women, in this condition.

Methods: We carried out two studies. In the first, case-control, study, we recruited men with and without NGU and examined their female partners for evidence of BV. The second, cohort design, study which ran concurrently with the first study involved recruiting women with and without BV and examining their male partners for evidence of NGU. The diagnoses of both NGU and BV were made microscopically to include symptomatic and asymptomatic individuals in both disease categories.

Results: In the case-control study 51 couples were recruited. Of these 39 men had NGU and 12 $(31 \%)$ of their female contacts had BV. In contrast, of 12 men without NGU, only one (8\%) of the female partners had BV (odds ratio $4 \cdot 89,95 \%$ CI: $0 \cdot 51-42 \cdot 27$ ). When only Chlamydia trachomatis negative patients were considered, the odds ratio for an association between BV and NGU was increased to $6 \cdot 77,95 \%$ CI: $0 \cdot 73-62 \cdot 68)$. Thirty eight couples were recruited to the cohort design study. Of 17 women with BV, $12(71 \%)$ of their male partners had NGU. In contrast, of 21 women without BV, seven (33\%) of their male partners had NGU ( $p=0.049$, odds ratio $4 \cdot 8$ ). When only $C$ trachomatis negative patients were considered, the significance of the association was increased $(p=0.037$; odds ratio 5.42).

Conclusions: An association exists between NGU and BV, and vice versa. If BV arises de novo the findings could help to explain the development of urethritis in stable sexual relationships.

(Genitourin Med 1997;73:373-377)

Keywords: non-gonococcal urethritis; bacterial vaginosis

\author{
Medical Research \\ Council Sexually \\ Transmitted Diseases \\ Research Group, \\ Department of \\ Genitourinary \\ Medicine, Jefferiss \\ Wing, St Mary's \\ Hospital, London W2 \\ F E A Keane \\ B J Thomas \\ D Taylor-Robinson \\ The Academic \\ Department of Public \\ Health, Imperial \\ College School of \\ Medicine, St Mary's \\ Hospital, London W2 \\ L Whitaker \\ A Renton \\ Correspondence to: \\ Dr Frances Keane, The City \\ Hospital, Department of \\ Genitourinary Medicine, \\ Infirmary Hill, Truro, \\ Cornwall TR1 $2 \mathrm{HZ}$. \\ Accepted for publication \\ 24 July 1997
}

\section{Introduction}

Urethritis is the most common condition seen in men attending genitourinary medicine (GUM) clinics in the United Kingdom. In the 1880 s, with the isolation of Neisseria gonorrhoeae and the development of the Gram stain, it became possible to differentiate between gonococcal and non-gonococcal urethritis (NGU). However, subsequent attempts to determine the aetiology of NGU have proved difficult. The role of Chlamydia trachomatis has become undisputed, this micro-organism having been isolated from up to $50 \%$ of cases of NGU in numerous studies. ${ }^{1-3}$ The evidence for the involvement of Ureaplasma urealyticum organisms (ureaplasmas), urethral colonisation of which is determined largely by sexual experience, ${ }^{4}$ is less clear. Thus, ureaplasmas have been isolated with similar frequency from men with NGU and from matched controls, ${ }^{5}$ but intraurethral inoculation with ureaplasmas has produced urethritis in human volunteers. ${ }^{6}$ Differential antibiotic trials have also supported a pathogenic role for ureaplasmas in a proportion of cases of NGU. ${ }^{78}$ Difficulties in isolating Mycoplasma genitalium by culture methods have previously hindered the investigation of the role of this organism in NGU. However, the recent development of DNA probes and particularly polymerase chain reaction (PCR) technology for detection has facilitated such research. In a number of studies, employing this technology, $M$ genitalium has been detected more frequently in men with $C$ trachomatis negative rather than $C$ trachomatis positive NGU. ${ }^{9-11}$ Trichomonas vaginalis may account for a small proportion of cases of NGU. Nevertheless, even when all of the aforementioned organisms are considered, the aetiology of NGU in a considerable proportion of patients with the disease remains unaccounted for. This led us to examine the possibility that the bacteria involved in bacterial vaginosis (BV) might be responsible for some cases. $\mathrm{BV}$ is the most common cause of abnormal vaginal discharge and a prevalence of between $24 \%$ and $37 \%$ has been reported in female attendees at sexually transmitted disease clinics. ${ }^{12}$ In BV, which is often asymptomatic, the vaginal ecology is altered with the normal Lactobacillus spp dominated flora being replaced by a predominantly anaerobic flora which includes Gardnerella vaginalis, $\mathrm{Myco-}$ plasma hominis, Mobiluncus spp, and Bacteroides spp. It was our hypothesis that some of the abnormal flora could be transferred to a male partner and be capable of causing NGU. As the first step in examining this proposal, we attempted to determine whether NGU in men was associated with the occurrence of $\mathrm{BV}$ in their partners. 


\section{Patients and methods}

Patients were recruited from among routine attendees at the GUM department in the Jefferiss Wing of St Mary's Hospital, Paddington, London. We undertook two studies: the first was a case-control study in which men with and without NGU were recruited and their female partners examined for evidence of BV. The second study was cohort designed and ran concurrently with the first, women with and without BV being recruited and their male partners examined for evidence of NGU. The diagnoses of both NGU and BV were made microscopically so that symptomatic and asymptomatic individuals were included in both disease categories. In men, NGU was diagnosed on the finding of $\geqslant 5$ polymorphonuclear leucocytes (PMNLs) per high power field $(\mathrm{HPF} ; \times 1000)$ of a Gram stained urethral smear, or $\geqslant 10$ PMNLs per HPF of a Gram stained thread from a first pass urine (FPU) sample. The control group consisted of men who did not have typical symptoms or signs of urethritis. They had $<5$ PMNLs per HPF of a Gram stained urethral smear and any threads present contained $<10$ PMNLs per HPF. In women, BV was diagnosed on the appearance of a Gram stained vaginal smear using a modification of Spiegel's criteria for diagnosis as described by Hay et $a l^{13}$ : that is, grade I, normal vaginal flora-predominantly lactobacillus morphotypes; grade II, intermediate-mixed lactobacillus and other bacterial morphotypes; and grade IIIfew or absent lactobacillus morphotypes but greatly increased numbers of $G$ vaginalis and other bacterial morphotypes, considered to represent unquestionable $\mathrm{BV}$. Both grade II and grade III flora were considered to be abnormal vaginal flora. Recruitment procedures for the studies were as follows. In the case-control study any heterosexual man attending the clinic who had not received antibiotics within the month before attendance and whose female partner would, in theory be able to attend the clinic, was invited to participate as an index patient. Men with microscopic evidence of NGU were assigned to the study group and those without to the control group. Any man with a history strongly suggestive of urethritis but with no microscopic evidence of same on either the first clinic visit or on repeat testing, having held his urine overnight was excluded. Control patients comprised mainly asymptomatic men attending for a check up or those with genital warts. Heterosexual women who had not received antibiotics in the previous month and whose male partner might be persuaded to attend the clinic were invited to enrol as index patients in the cohort study. Depending on the appearances of the Gram stained vaginal smear, they were assigned to either the study (grade II or III) or control group (grade I). Partners of index patients in both studies were recruited as follows. If the partner was present in the clinic at the time of recruitment of the index case then they were invited to participate in the study. If they were not present then the index patient was handed a letter to give to the partner inviting him or her to attend. Gonorrhoea was diagnosed by microscopy and culture of urethral samples from men and urethral and cervical samples from women. Couples in which either partner had gonorrhoea were excluded. Also, any woman in the cohort study who had Trichomonas vaginalis or who had Candida spp together with BV detected by vaginal smear microscopy was excluded along with her partner. After collection of the urethral smear from men, and before collection of the FPU sample, a nasopharyngeal swab (MW 142, Medical Wire and Equipment $\mathrm{Co}$ ) was passed $2-4 \mathrm{~cm}$ into the urethra, rolled over a MicroTrak slide (Syva, UK) and the smear fixed in acetone to be used for the detection of $C$ trachomatis. In women after collection of the vaginal smear for Gram staining to determine the grade of vaginal flora and to document the presence or absence of Candida spp, a sample from the posterior fornix, obtained with a plastic loop, was mixed with a drop of water and the resultant "wet preparation" examined under polarised light for the presence of $T$ vaginalis. A $5 \mathrm{~mm}$ cotton tipped swab (MW 102, Medical Wire and Equipment Co) was used to collect an endocervical sample which was rolled over a MicroTrak slide and fixed as before for the detection of $C$ trachomatis. Ten to $20 \mathrm{ml}$ of FPU were also obtained. Cervical and urethral smears were stained with MicroTrak DFA direct specimen test reagent 3 (Syva, UK) as described previously. ${ }^{14}$ Specimens containing one or more elementary bodies (EBs) were considered to be positive for $C$ trachomatis. Each urine sample was thoroughly mixed and a $1 \mathrm{ml}$ sample was centrifuged at $13000 \mathrm{rpm}$ for 10 minutes in a MicroCenatur centrifuge (MSE). The deposit was resuspended in 100 $\mu \mathrm{l}$ of phosphate buffered saline and $20 \mu \mathrm{l}$ of this was dried on to a MicroTrak slide, fixed in acetone, and stained as above. Samples containing one or more EBs were considered to be positive. In our hands, the DFA test has a sensitivity equal to that of amplified DNA methods. All patients completed a questionnaire which sought information about age, ethnic origin, symptomatology, recent antibiotic usage, and sexual history. Ethics committee approval was obtained before study commencement. The data were analysed using SAS statistical software. Fisher's exact test was used to calculate $\mathrm{p}$ values for group differences in proportions and the Kruskall Wallis $\chi^{2}$ test for group differences in the distribution of continuous variables. Confidence interval on univariate odds ratios and relative risks were calculated using standard methods on a log transformed scale. ${ }^{15}$

\section{Results}

The case-control study comprised 51 couples, 39 men with NGU and 12 men without NGU, and their respective female partners. The "failure of partner to participate" rates were, for men with NGU, 37/76 (49\%) and for controls seven $(37 \%)$ of 19 . Logistic regression analysis identified no association between known char- 
Table 1 Case-control study-relation between non-gonococcal urethritis(NGU) and bacterial vaginosis $(B V)$

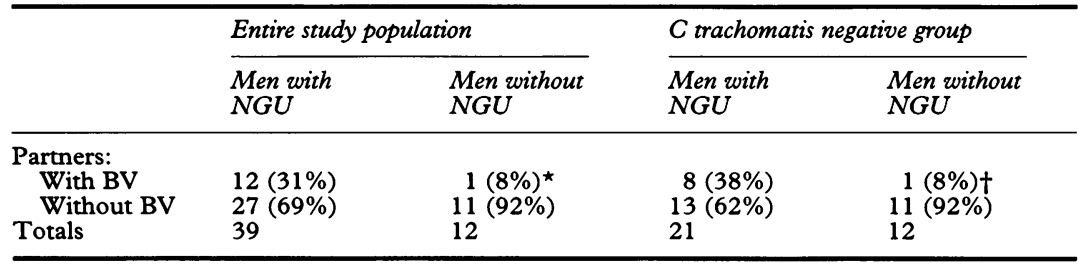

*Odds ratio $=4 \cdot 89, \mathrm{CI}: 0 \cdot 51-42 \cdot 27, \mathrm{p}=0 \cdot 15$.

tOdds ratio $=6 \cdot 77, \mathrm{CI}: 0 \cdot 73-62 \cdot 86, \mathrm{p}=0 \cdot 11$.

Grades of vaginal flora in partners of:

$\begin{array}{llll}\text { cases } & \text { I: } 27(69 \%) & \text { II: } 2(5 \%) & \text { III: } 10(26 \%) \\ \text { controls } & \text { I: } 11(92 \%) & \text { II: } 0 & \text { III: } 1(8 \%)\end{array}$

acteristics of index cases (age, ethnicity, and parameters of sexual behaviour) and the participation of partners. The men and women in the NGU group tended to be younger than their counterparts in the control group, the median age of men with NGU being 30.5 years and that of men without NGU being 32 years $\left(p=0.046, \mathrm{KW} \chi^{2}=3.97\right)$. Female partners of men with NGU had a median age of 28 years while that of partners of male controls was 31.5 years $\left(\mathrm{p}=0.034, \mathrm{KW} \chi^{2}=\right.$ $4 \cdot 47)$. There was no significant difference in the ethnicity of the cases and controls. The relation between NGU and abnormal vaginal flora in the female partner, considering first the entire group and then only couples in whom neither partner had $C$ trachomatis, is shown in table 1 . Only two partners had grade II flora, so that for analysis these have been combined with the 10 who had grade III flora and all are discussed as BV henceforth. There was a trend for partners of men with NGU to have BV more often than those of men without NGU-namely in the entire group, $12(31 \%)$ compared with one $(8 \%)$ ( $p=0 \cdot 15$, odds ratio $4 \cdot 89$; 95 \% CI: $0 \cdot 51-42 \cdot 27)$. $C$ trachomatis was detected only in couples in which the man had NGU; in the man alone in eight couples, in both partners in six cases, and in the woman alone in four couples, giving an overall detection rate of $36 \%$ in the 39 men with NGU. In the $C$ trachomatis negative sample comprising 21 men with NGU (of whom 13 (62\%) were symptomatic) and their partners, eight (38\%) of their female partners had BV in comparison with one $(8 \%)$ partner of men without NGU, and the association between NGU and BV was slightly stronger than before, with an odds ratio of $6 \cdot 77$ ( $\mathrm{p}=0 \cdot 11,95 \% \mathrm{CI}$ : $0 \cdot 73-62 \cdot 80)$. In addition, logistic regression models were used to control for the potentially confounding

Table 2 Cohort study: relation between bacterial vaginosis (BV) and non-gonococcal urethritis (NGU)

\begin{tabular}{|c|c|c|c|c|}
\hline & \multicolumn{2}{|c|}{ Entire study population } & \multicolumn{2}{|c|}{$C$ trachomatis negative group } \\
\hline & $\begin{array}{l}\text { Women with } \\
B V\end{array}$ & $\begin{array}{l}\text { Women without } \\
B V\end{array}$ & $\begin{array}{l}\text { Women with } \\
B V\end{array}$ & $\begin{array}{l}\text { Women without } \\
B V\end{array}$ \\
\hline \multicolumn{5}{|l|}{ Partners: } \\
\hline $\begin{array}{l}\text { With NGU } \\
\text { Without NGU } \\
\text { Totals }\end{array}$ & $\begin{array}{r}12(71 \%) \\
5(29 \%)^{\star} \\
17\end{array}$ & $\begin{array}{l}7(33 \%) \\
14(67 \%) \\
21\end{array}$ & $\begin{array}{c}10(71 \%) \\
4(29 \%) \dagger \\
14\end{array}$ & $\begin{array}{l}6(32 \%) \\
13(68 \%) \\
19\end{array}$ \\
\hline
\end{tabular}

${ }^{\star} \mathrm{p}=0 \cdot 049$, odds ratio $=4 \cdot 80,95 \% \mathrm{CI}: 1 \cdot 20-19 \cdot 20$.

relative risk $=2 \cdot 12,95 \% \mathrm{CI}: 1 \cdot 08-4 \cdot 17$.

tp $=0.037$, odds ratio $=5 \cdot 41,95 \%$ CI: $1 \cdot 20-24 \cdot 52$

relative risk $=2 \cdot 26,95 \%$ CI: $1 \cdot 08-4 \cdot 74$.

Grades of vaginal flora:

cases

I: 0

II: $2(11 \%)$

III: $15(89 \%)$

III: 15 effects of age, ethnicity, and parameters of sexual behaviour. Estimates of odds ratios were almost identical to those estimated from the univariate analysis shown in table 1 . The cohort designed study comprised 38 women, 17 with abnormal vaginal flora, 21 with normal vaginal flora, and their respective male partners. As only two women had grade II flora, they have been combined with the 15 who had grade III flora and, as before, all are discussed as BV. Seventy eight (82\%) of 95 male partners of women with BV failed to attend, as did nine $(30 \%)$ of the partners of control women. Logistic regression analysis identified no association between known characteristics of index cases (age, ethnicity, and parameters of sexual behaviour) and the participation of partners. There was no appreciable difference in age or ethnicity between the study and control populations. The relation between BV and NGU in the entire group and in the subset of couples who were both $C$ trachomatis negative is shown in table 2 . In the entire group, $12(71 \%)$ of the male partners of women with BV had microscopic evidence of NGU compared with seven $(33 \%)$ of the male partners of women who did not have BV ( $\mathrm{p}=$ 0.049 , odds ratio $=4 \cdot 80,95 \%$ CI: $1 \cdot 20$ 19.20). Examination of the $C$ trachomatis negative subset showed similar results $(\mathrm{p}=0.037$, odds ratio $=5 \cdot 41 ; 95 \%$ CI: $1 \cdot 20-24 \cdot 52)$. Further analysis of the $C$ trachomatis negative group from which symptomatic men with NGU (six $(37 \cdot 5 \%)$ ) were excluded, revealed that seven $(70 \%)$ male partners of women with BV had NGU, compared with only three $(20 \%)$ partners of women with normal flora $(\mathrm{p}=0.034$, odds ratio $=9.33 ; 95 \% \mathrm{CI}$ : 1.47-59.48). As before, logistic regression models were used to control for the potentially confounding effects of age, ethnicity, and parameters of sexual history. Estimates of odds ratios were almost identical to those obtained from the univariate analysis shown in table 2 .

\section{Discussion}

There have been a few previous attempts to examine the role that some of the organisms associated with BV might have in the aetiology of NGU. In this regard, $M$ hominis itself does not appear to be a promising candidate since it has been found more frequently in men who did not have NGU than in those who did. ${ }^{16}$ There are reasons, as mentioned before, for associating ureaplasmas with some cases of NGU. Whether this might arise from the association of ureaplasmas with BV is not clear, although they would appear to be associated with BV less than are $M$ hominis organisms (Rosenthein et al, unpublished data). Some workers have isolated Bacteroides ureolyticus from men with NGU more often than from those without the disease, interestingly in those with both $C$ trachomatis and $U$ urealyticum negative NGU. ${ }^{17}$ In addition, the results of a double blind, placebo controlled trial of the use of metronidazole in $C$ trachomatis negative NGU seemed to lend support for 
the aetiological role of $B$ ureolyticus in this condition. Of $23 C$ trachomatis negative, anaerobe positive men, seven $(78 \%)$ of nine responded clinically to metronidazole compared to one (7\%) of 14 receiving a placebo. In addition, only six $(23 \%)$ of 26 anaerobe negative men responded to metronidazole therapy. ${ }^{18}$ However, other workers have isolated $B$ ureolyticus as frequently from men with NGU as from those without NGU and contend that this organism is merely a part of the normal urethral flora. ${ }^{19} G$ vaginalis has been isolated from the urethra of $11.4 \%$ of male GUM attendees and more often from heterosexual than homosexual individuals. ${ }^{20}$ Its presence, however, was not associated with symptoms of urethritis, although microscopic evidence of inflammation was not sought. Further evidence for passive urethral acquisition by men of BV associated organisms, including Mobiluncus spp, $M$ hominis, and $G$ vaginalis, has been provided by culturing genital samples from the male partners of women with BV. ${ }^{21}$ However, it was also observed in this study, in which clinical disease was not assessed, that urethral colonisation ceased within several weeks of condom usage for vaginal intercourse. Other workers have attempted to determine whether there is an association between NGU and BV. Mitchell et al, ${ }^{22}$ studying NGU in the context of other genitourinary infections, examined 52 female contacts of 41 men with NGU and found BV in 17 of them. This contrasted with a finding of $\mathrm{BV}$ in five of 30 female contacts of 46 men without NGU. However, as the nature of the pairings was not detailed it was difficult to assess the strength of any possible association in this study. Arumainayagam et $a^{23}$ found NGU in 30 of 100 male partners of women with BV. However, the control group in this study consisted of heterosexual men who had partners the BV status of whom was unknown, so that a comparison of the two groups was impossible. In contrast, the results of our two complementary studies suggest an association between NGU and BV, although statistical significance was reached only in the cohort study. It was estimated that the case-control study would require recruitment of couples for a further 12 months to ensure adequate numbers of controls to demonstrate a similar association, which was not a practical proposition. Interestingly, the association in both studies was independent of the presence of $C$ trachomatis. Although the number of men with asymptomatic NGU in the case-control study was too small to permit statistical analysis, the association between NGU and BV was strongest in the cohort study when men with symptomatic NGU were excluded from the analysis of the $C$ trachomatis negative group. The numbers in the latter analysis are small but it is apparent that despite at least three quarters of the men in both the study and control groups having been in a monogamous relationship for a minimum of 3 months, the majority of those whose female partners had BV had NGU. The possibility that the findings of both studies may have arisen through sampling bias needs to be considered given the substantial proportion of index cases for whom it did not prove possible to examine a partner because of failure to attend. It is plausible that some partners in either study might have been more inclined to attend if the index case had a diagnosed genitourinary condition than if they were a control, because of anxiety, in the former case that they might have been affected. Similarly, partners might have been more inclined to attend if they themselves had symptoms suggestive of a genitourinary condition. However, in both these events, the observed association would remain unaffected as long as any increased tendency to attend was not correlated with both the index case's and the partner's disease status simultaneously. If, for instance, the probability of attendance of the partner of a woman with $\mathrm{BV}$, who himself had NGU, was more likely than that of a partner of a woman without BV, who himself had NGU, then the association reported could clearly have arisen through bias. Although this particular scenario is unlikely because most of the men in the cohort study who had NGU were asymptomatic, the possibility of bias could only be excluded conclusively by achieving much higher rates of successful partner recruitment (which we do not believe to be possible in inner London clinics) or through the use of non-observational research designs. Interestingly, the proportion of $C$ trachomatis negative men with asymptomatic NGU in the cohort study is considerable, the very nature of which makes its clinical relevance difficult to determine. Obviously, there are three possible outcomes: persistence of asymptomatic NGU indefinitely, resolution of the microscopic evidence of NGU, or development of clinical urethritis. Follow up of patients was not possible so that the outcome is unknown. The more stable sexual relationships of the cohort study make the findings easier to interpret than those of the case-control study in which about half of the study group had changed sexual partners in the previous 3 months. Also, as it was mainly regular partners of men in the case-control study who attended, it could be argued that an incomplete picture of the microbiology likely to be involved in the aetiology of NGU in these patients has been obtained. It could also be argued that our diagnostic criteria for NGU-that is, $\geqslant 5$ PMNLs per HPF of a urethral smear or $\geqslant 10$ PMNLs per HPF of a thread from a FPU specimen, were too over inclusive for NGU. However, the results of several studies have demonstrated that $\geqslant 5$ PMNLs per HPF of a urethral smear is indicative of significant NGU. ${ }^{24} 25$ The situation regarding pyuria is less clear since most investigators to date have used a sediment obtained by centrifugation of a FPU specimen and opinion varies as to whether 10 or 15 PMNLs per HPF is a suitable cut off point. ${ }^{25}{ }^{26}$ However, this procedure is not practical in a busy GUM clinic where it is standard policy to examine threads obtained from a FPU for PMNLs. In any case, only five men in the entire study had NGU diagnosed on $\geqslant 10$ PMNLs per HPF in a thread of a 
FPU. For the remainder, the diagnosis was based on finding at least 20 PMNLs per HPF in the thread or on the examination of a urethral smear. Despite the caveats above, it appears that an association does exist between NGU and BV and that it is independent of $C$ trachomatis. This association, if it implies that BV causes NGU, has important implications as it means that NGU can arise within the context of a monogamous relationship and challenges the view that it should always be regarded as a sexually transmitted disease involving a third party. We can only speculate at present as to the mode of action of BV associated organisms in the male urethra. Classically, BV does not cause an inflammatory reaction within the vagina but evidence has accumulated for its association with preterm labour, late miscarriage, and postpartum endometritis, ${ }^{13} 27$ suggesting that it can be linked to inflammation elsewhere. An immunological response to the bacteria associated with BV or a direct effect of toxins released from organisms such as Mobiluncus $\mathrm{spp}^{28}$ could be responsible for the development of an inflammatory reaction within the male urethra. More detailed microbiological assessments are required and our findings need to be substantiated, ideally by repeating our study in a more stable clinic population where recruitment of partners is less difficult and where longitudinal follow up, especially of male partners of women with BV is more feasible. This may help to clarify the outcome of asymptomatic NGU and determine further management policy in terms of treatment and follow up of such patients.

The authors would like to thank Dr Elizabeth Claydon for permitting access to the patients attending the Jefferiss Wing and Dr Cathy Ison for her advice regarding the interpretation of Gram stained vaginal smears. This project was funded by a grant from the Medical Research Council.

1 Oriel JD, Reeve P, Wright JT, Owen J. Chlamydial infection of the male urethra. $B r \mathcal{F}$ Vener Dis 1976;52:46-51.

2 Bowie WR, Wang S-P, Alexander ER, Floyd J, Forsyth PS, Pollock HM, et al. Etiology of nongonococcal urethritis. Evidence for Chalmydia trachomatis and Ureaplasma urealyticum. $\mathcal{F}$ Clin Invest 1977;59:735-42.

3 Perroud HM, Miedzybrodzka K. Chlamydial infection of the urethra in men. Br f Veneral Dis 1978;54:45-9.

4 McCormack WM, Lee Y-H, Zinner SH. Sexual experience and urethral colonization with genital mycoplasmas. study in normal men. Ann Intern Med 1973;78:696-8.

5 Lee Y-H, Tarr PI, Schumacher JR, Rosner B, Alpert S, McCormack WM. Re-evaluation of the role of $\mathrm{T}$ mycoplasmas in non-gonococcal urethritis. Sex Transm

6 Taylor-Robinson D, Csonka GW, Prentice MJ. Human intra-urethral inoculation of ureaplasmas. $Q \mathcal{F ~ M e d ~ N e w ~}$ Series 1977; 46:309-26.

7 Bowie WR, Alexander ER, Floyd JF, Holmes J, Miller Y, Holmes KK. Differential response of chlamydial and ureaplasma-associated urethritis to sulphafurazole (sulfisoxazole) and aminocyclitols. Lancet 1976;ii:1276-8.

8 Coufalik ED, Taylor-Robinson D, Csonka GW. Treatment of nongonococcal urethritis with rifampicin as a means of defining the role of Ureaplasma urealyticum. Br $\mathcal{f}$ Vener defining the role of
Dis $1979 ; 55: 36-43$.

9 Horner PJ, Gilroy CB, Thomas BJ, Naidoo ROM, TaylorRobinson D. Association of Mycoplasma genitalium with Robinson D. Association of Mycoplasma genitalium with
acute non-gonococcal urethritis. Lancet 1993;342:582-5.

10 Jensen JS, Orsum R, Dohn B, Uldum S, Worm A-M, Lind K. Mycoplasma genitalium: a cause of male urethritis? Genitourin Med 1993;69:265-9.

11 Deguchi T, Komeda H, Yasuda M, Tada K, Iwata $H$, Asano $\mathrm{M}$, et al. Mycoplasma genitalium in non-gonococcal urethritis. Int $\mathcal{F} S T D$ AIDS 1995;6:144-5.

12 Hay PE, Taylor-Robinson D. Diagnosis of bacterial vaginosis in a gynaecology clinic. Br f Obstet Gynaecol 1992; 99:63-6.

13 Hay PE, Lamont RF, Taylor-Robinson D, Morgan J, Ison CA, Pearson J. Abnormal bacterial colonisation of the genital tract and subsequent preterm delivery and late genital tract and subsequent preterm
miscarriage. $B M \mathcal{F} 1994 ; 308: 295-8$.

14 Thomas BJ, Evans RT, Hawkins DA, Talyor-Robinson D. The sensitivity of detecting Chlamydia trachomatis elementary bodies in smears by use of a fluorescin labelled monoclonal antibody: comparison with conventional chlamydial isolation. $\mathcal{F}$ Clin Pathol 1984;37:812-6.

15 Rothman KJ. Logistic regression models where used to control for measured potential confounders. Modern epidemiology. Boston: Little Brown, 1986:144.

16 Holmes KK, Handsfield $\mathrm{HH}$, Wang SP, Wentworth BB, Turck $M$, Anderson JB, et al. Etiology of nongonococcal Turck M, Anderson JB, et al. Etiology of nongo
urethritis. N Engl f Med 1975;292:1199-205.

17 Fontaine EA, Taylor-Robinson D, Hanna NF, Coufalik ED. Anaerobes in men with urethritis. Brf Vener Dis 1982;58:321-6.

18 Hawkins DA, Fontaine EAR, Thomas BJ, Boustouller YL, Taylor-Robinson D. The enigma of non-gonococcal urethritis: role for Bacteroides ureolyticus. Genitourin Med 1988;64:10-3.

19 Woolley PD, Kinghorn GR, Talbot MD, Duerden BI. Microbiological flora in men with non-gonococcal urethritis with particular reference to anaerobic bacteria. Int $f$ STD AIDS 1990;1:122-5.

20 Dawson SG, Ison CA, Csonka G, Easmon CSF. Male carriage of Gardnerella vaginalis. Br $\mathcal{F}$ Vener Dis 1982;58: 243-5.

21 Holst E. Reservoir of four organisms associated with bacterial vaginosis suggests lack of sexual transmission. $\mathcal{F}$ Clin Microbiol 1990;28:2035-9.

22 Mitchell SA, Shukla SR, Thin RN. Aetiology of non-gonococcal urethritis: a possible relation to other infections. Int $\mathcal{F}$ STD AIDS 1990;1:429-31.

23 Arumainayagam JT, de Silva Y, Shahmanesh M. Anaerobic vaginosis: study of male sexual partners. Int $\mathcal{f} S T D$ AIDS $1991 ; 2: 102-4$.

24 Swartz SL, Kraus SJ, Herrmann KL, Stargel MD, Brown WJ, Allen SD. Diagnosis and etiology of nongonococcal urethritis. F Infect Dis 1978;138:445-54.

25 Bowie WR. Comparison of Gram stain and first-voided urine in the diagnosis of urethritis. Sex Transm Dis 1978; 5:39-42.

26 Desai K, Robson HG. Comparison of the Gram-stained urethral smear and first- voided urine sediment in the diagnosis of nongonococcal urethritis. Sex Transm Dis 1982;9:21-5.

27 Rosene KA, Eschenbach DA, Tompkins LS, Kenny GE, Watkins $\mathrm{H}$. Polymicrobial early postpartum endometritis with facultatively anaerobic and anaerobic bacteria, genital mycoplasmas and Chlamydia trachomatis; treatment with piperacillin or cefoxitin. F Infect Dis 1986;153 1028-37.

28 Taylor-Robinson AW, Borriello SP, Taylor-Robinson D. Identification and preliminary characterization of a cytotoxin isolated from Mobiluncus spp. Int $f$ Exp Pathol toxin isolated from 